\title{
Théologiques
}

\section{La foi chrétienne, ressource pour vivre les questions de société}

\section{Geneviève Médevielle}

Volume 14, numéro 1-2, automne 2006

Les lieux de la théologie aujourd’hui

URI : https://id.erudit.org/iderudit/014312ar

DOI : https://doi.org/10.7202/014312ar

Aller au sommaire du numéro

Éditeur(s)

Faculté de théologie et de sciences des religions, Université de Montréal

ISSN

1188-7109 (imprimé)

1492-1413 (numérique)

Découvrir la revue

Citer cet article

Médevielle, G. (2006). La foi chrétienne, ressource pour vivre les questions de société. Théologiques, 14(1-2), 81-106. https://doi.org/10.7202/014312ar

\section{Résumé de l'article}

Pour répondre aux grands défis de la société actuelle, la foi chrétienne peut-elle nous aider à inventer, orienter et organiser une vie morale? À la lumière de la confrontation de l'Église de France à la délicate question de l'accueil des étrangers illégaux, l'auteure revisite les trois modèles d'éthique théologique disponibles dans les débats postconciliaires: l'éthique autonome, l'éthique de la foi et l'éthique communautarienne de la vertu. Forces et limites de ces modèles d'implications sociales de la foi supposent la catégorie de l'intégralisme de la foi.
Tous droits réservés (C Faculté de théologie et de sciences des religions, Université de Montréal, 2006
Ce document est protégé par la loi sur le droit d'auteur. L'utilisation des services d'Érudit (y compris la reproduction) est assujettie à sa politique d'utilisation que vous pouvez consulter en ligne.

https://apropos.erudit.org/fr/usagers/politique-dutilisation/ 


\title{
La foi chrétienne, ressource pour vivre les questions de société
}

\author{
Geneviève MÉDEVIELLE \\ Faculté de théologie et de sciences religieuses \\ Institut Catholique de Paris
}

\section{Introduction}

L'espace d'interrogation que je suis chargée d'ouvrir par ce texte concerne la possibilité de penser la foi chrétienne comme ressource pour vivre les questions de société. Cette question est familière à la théologienne moraliste que je suis. Elle l'est aussi au théologien en quête de fonder une théologie politique pour aujourd'hui. De fait, c'est une des questions fondamentales que la théologie contemporaine se doit de réfléchir. Mais c'est en moraliste que j'affronterai la question, puisqu'elle touche les terrains de l'éthique théologique et de ses modèles disponibles pour rendre compte de la pertinence de la foi dans nos sociétés pluralistes et sécularisées.

Depuis le concile de Vatican II, la théologie morale, dans ses dimensions tant fondamentale que sectorielle touchant à la vie sociale, n'a cessé de tenter de rendre raison à l'affirmation que la foi a des ressources pour résoudre les grands défis qu'affrontent nos sociétés. Mais elle l'a fait dans un contexte où l'on pouvait encore compter sur une certaine culture chrétienne. Or, aujourd'hui, le soupçon né avec l'Aufklärung sur le fait que le christianisme aurait fait son temps pèse à nouveau très fort en morale. Ce soupçon relayé par des philosophes tels que Alain Badiou, André ComteSponville ou Luc Ferry s'exprime de la façon suivante: les formes éthiques de la vie pourraient être complètement indépendantes des formations religieuses ${ }^{1}$. Il ne resterait alors au moraliste catholique que deux options, soit

1. Le débat entre Habermas et le théologien Jean-Baptiste Metz est à cet égard significatif (voir Habermas 1994). 
se rallier à l'éthique procédurale ${ }^{2}$ de nos sociétés sécularisées pour être résolument moderne, mais avec le risque d'y laisser sa spécificité, soit prendre la position réactionnaire et nostalgique d'une éthique communautarienne critique et contre-culturelle, mais alors ce serait d'avance avoir renoncé à engager un débat éthique avec la culture.

On peut percevoir que le positionnement par rapport à cette question engendre des manières de faire de la théologie qui ne sont pas anodines pour la définition même de la théologie. Si la théologie morale a pour vocation d'éclairer et de servir l'agir humain à partir de la foi, il importe de prendre au sérieux, d'une part, l'horizon culturel, politique et social dans lequel elle se pense: un espace public désormais pluraliste et, d'autre part, la foi même comme source originale d'éclairage du sens de la vie et des pratiques.

Mais cette question passionnante est difficile, car nous manquons cruellement de modèles adéquats pour penser la foi comme ressource telle que nous la vivons concrètement. J'en veux pour preuve une recherche que je mène depuis ma contribution à la rédaction en 1996 de la Lettre aux catholiques de France, adoptée par l'assemblée plénière des évêques de France, sur la proposition de la foi (voir Conférence des évêques de France 1996). Cette lettre était censée apporter aux communautés chrétiennes un outil pour entrer dans une intelligence évangélique de la situation de la foi et dire la tâche de l'Église dans la société à l'entrée du troisième millénaire. La bonne réception de cette lettre dans l'Église et la société en France vient de ce qu'elle présentait, au cœur d'une crise sans précédent de la transmission et de la culture, une figure de l'Église, ouverte au pluralisme et au dialogue, dépendante d'autrui et vulnérable, placée dans l'écoute et fidèle à la foi qui la constitue. Une Église qui a cessé de se penser comme l'institution autosuffisante dont l'appartenance se faisait à partir de la conformité à la définition objective et juridique de l'ecclésiologie sociétaire. Une Église qui, parce qu'elle interprète le présent comme chance, pense pouvoir contribuer au vouloir-vivre de notre société en faisant résonner les grandes affirmations de la foi : la valeur absolue

2. Les débats des comités d'éthique ont souligné qu'on ne pouvait pas se contenter d'une éthique « discuteuse appuyée à un relativisme moral ». Ils ont alors mis en valeur une éthique de délibération collective capable de désigner un noyau consensuel. Cette éthique procédurale trouve son origine dans les réflexions de Max Weber (Le savant et le politique) sur l'éthique de conviction et l'éthique de responsabilité. Aujourd'hui elle trouve une expression théorique dans l'éthique communicationnelle de J. Habermas. Des points de vue méthodologique et pragmatique, l'éthique procédurale centre le débat éthique sur la justification sociale des normes abandonnant aux convictions personnelles les critères de bien et de mal auxquels elle substitue ceux de right (convenable) et de wrong (non-convenable) pour l'individu ou le groupe. 
de toute personne humaine, l'option radicale pour les pauvres, la quête de l'amour, du pardon et de la réconciliation au cœur de la violence, jusqu'au delà du mal (Conférence des évêques de France 1994, 47-48).

Or, je me rends compte aujourd'hui que nous ne sommes sans doute pas allés assez loin dans la réponse à apporter à la question de la foi comme ressource dans notre société postmoderne. Nous osions écrire, à partir du déchiffrement des comportements croyants, que la foi a un réel impact sur la société. Mais nous le disions sous la modalité de la conviction: «Nous ne pouvons pas nous résigner à une totale privatisation de notre foi, comme si l'expérience chrétienne devait rester enfouie dans le secret des cœurs, sans prise sur le réel du monde et de la société. Notre Église n'est pas une secte. » (Conférence des évêques de France 1996, 34)

En ce qui me concerne, dans le concert du débat international des moralistes, partagés entre libéraux et communautariens, je n'arrivais pas à dégager l'originalité du positionnement de cette Église immergée dans un monde pluriel qui, sans renoncer aux meilleurs fruits de la Modernité, sait qu'elle ne peut renoncer à être une instance de discernement critique et de proposition. Les outils, modèles, catégories et théories que nous utilisons pour dire l'impact social de la foi chrétienne ne sont pas anodins. Marqués au sceau historique d'un contexte culturel et d'une philosophie politique qui ne s'énoncent pas toujours, ces outils peuvent nous priver de rendre compte adéquatement de l'expérience croyante ou peuvent nous faire généraliser indûment des données très contextuelles. C'est comme cela que je m'explique la difficile entrée en dialogue de la philosophie et de la théologie françaises avec les libéraux et les communautariens très marqués par le contexte nord-américain (voir Berten et al. 1997, 1-19).

La catégorie qui me paraît la plus apte à rendre compte du paradoxe d'une foi privatisée par la sécularisation, mais revendiquant un impact sur la société est celle de "l'intégralisme» de la foi. Reprise d'une catégorie classique en histoire et en sociologie des religions, la notion d'intégralisme de la foi a l'avantage de rejoindre ce que tout spirituel habitué au discernement des esprits sait, que la foi est «intégrale» parce qu'elle joue comme instance critique et principe de discernement dans tous les domaines de la pensée et de l'activité du croyant ${ }^{3}$. La définition de l'intégralisme de la foi,

3. La foi est intégrale parce que:

... la vie, la vie humaine, la mienne et celle du monde, prennent un sens renouvelé lorsqu'on fait le choix de croire chrétiennement. La vie et le monde ne sont en effet alors plus aussi opaques qu'ils apparaissaient l'être auparavant. À plus forte raison ne sont-ils pas absurdes: nous allons quelque part, nous sommes attendus, il y a une Maison, l'espérance est possible, et pour éternellement. (Doré 2003, 127) 
si on la prend à partir de ce versant proprement théologique, est paradoxale. On a pris en effet l'habitude, dans la société sécularisée, de lier historiquement intransigeantisme et intégralisme ${ }^{4}$. Or, les réalités que recouvrent ces deux termes ne sont pas identiques.

L'intransigeantisme traditionnel, nous dit le sociologue Jean-Marie Donégani, trouvait sa source dans cette auto-compréhension de l'Église comme société parfaite, inégale et hiérarchique et dans cette perception de la modernité comme empoisonnée par l'idée d'autonomie de la raison et de la séparation des sphères. L'intégralisme d'un autre côté, c'est cette conviction que l'essence de la foi chrétienne est de concerner toute la vie de l'homme, d'embrasser toutes les dimensions de son être et non pas seulement l'activité rituelle ou les croyances concernant le sacré reléguées dans la sphère privée. $(1999,52)$

Pour montrer à quelles conditions cette catégorie de la foi intégrale peut nous aider à penser la capacité de la foi à inventer, orienter et organiser une vie morale et politique je partirai, en bonne moraliste, d'un cas. Il s'agira de la confrontation de l'Église de France à la délicate question de l'accueil des étrangers illégaux sur le territoire français. Cet exemple a le mérite d'exposer la situation paradoxale d'une Église minoritaire dans la société postmoderne qui, contrairement à toutes les attentes de l'Aufklärung, se risque encore, au nom de sa foi, à une parole publique et à des actions dans le champ social et politique. À la lumière de cette expérience de la foi vécue, j'examinerai les trois modèles d'éthique théologique disponibles dans les débats postconciliaires: l'éthique autonome, l'éthique de la foi et l'éthique communautarienne de la vertu. J'évaluerai leurs forces et leurs limites pour rendre compte des implications sociales d'une foi intégrale.

\section{La foi à l'œuvre dans l'accueil de l'étranger dans l'Église de France}

\subsection{Une situation conflictuelle entre la mission de l'Église et la société politique}

Commençons par constater que, totalement absente du débat public il y a trente ans, la question de l'immigration et de l'accueil de l'étranger a surgi

4. Le catholicisme de la fin du XIX ${ }^{\mathrm{e}}$ siècle, en lutte constante avec la société libérale et sa philosophie, s'exprime très clairement dans les encycliques de Léon XIII et dans celle de Pie X, E supremi apostolatus (Pie X s.d., 35s.) quand l'Église se comprend comme une contre-société engagée dans une lutte finale et apocalyptique avec la société moderne et dessine l'utopie intégrale d'un ordre nouveau opposé au libéralisme et au socialisme. 
dans l'actualité française pour s'affirmer comme un enjeu politique et social de première importance. Il n'est pas exagéré de dire que la question est devenue le symptôme des angoisses du pays en ce temps de crise. Que l'on parle du malaise des banlieues, de la difficile intégration de certaines communautés maghrébines, de l'échec scolaire, du chômage, de la paupérisation d'un million d'enfants en France, de la montée de l'islam, du port du foulard par les jeunes filles musulmanes, de la montée du racisme, des succès du Front National, on croise toujours la question de l'accueil de l'étranger comme "problème" social et politique. Le débat public longtemps cantonné dans des oppositions simplistes entre partis de gauche et de droite, entre générosité et répression, entre naïveté et réalisme, entre responsabilité et conviction s'est élargi aux religions. Il reste très passionnel, car l'accueil de l'étranger est vécu comme une épreuve.

Le débat public est d'autant plus vif que tout rôle public et toute fonction sociale d'une religion sur le territoire français sont régis par le principe de laïcité. La laïcité, comprise au sens de Jaurès et des pères fondateurs de la III ${ }^{\mathrm{e}}$ République, tend à sceller un accord social sur un certain nombre de valeurs fondamentales pour le vivre-ensemble en s'interdisant de les réduire à une quelconque univocité philosophique ou religieuse. Elle est de fait compatible avec l'accueil dans l'espace public de religions ou de spiritualités faisant appel à des données extérieures à la société civile, car la laïcité admet le pluralisme. D'un point de vue positif, elle peut alors ouvrir des possibilités aux croyances, parce qu'elle implique le partage de l'espace public et la confrontation, au sein de cet espace, de différentes formes de convictions concernant tous les aspects de la vie. Elle peut aussi offrir à chacun la possibilité de se frayer sa propre voie éthique en n'étant tenu par aucun système de prescription particulier et en conjuguant à sa guise les valeurs ou intuitions positives venant des divers groupes présents dans l'espace public. Mais dans le même temps, et cette fois-ci de manière négative, on ne saurait nier que, tout en élargissant les horizons et en favorisant le pluralisme, la laïcité crée les conditions d'une crise fondationnelle des valeurs, qui se trouvent déliées de tout ordre spirituel commun. De fait, la libération des croyances et de l'éthique de toutes formes de disciplines imposées n'est possible qu'à partir de l'invitation à ne pas mélanger spirituel et politique.

La crise est encore plus grave quand cette démarche laïque est conjuguée à celle de la sécularisation de la société, qui refuse d'en appeler à des registres de légitimité qui ne sont pas ceux selon lesquels fonctionne la 
société civile et politique. Les croyances religieuses sont exclues de la sphère publique et remises à la conviction privée. Seule la raison publique qui préside à la sélection des arguments éthiques admis dans la société peut définir les normes nécessaires au vivre-ensemble.

C'est en fonction de cette place de la religion au sein de la société française que depuis une dizaine d'années l'Église de France est confrontée directement à la question de l'accueil de l'étranger. Elle y est confrontée par de nombreuses grèves de la faim qui se sont déroulées dans des locaux d'Église ${ }^{5}$, par des occupations d'églises et par la question que se sont posée des communautés chrétiennes: pouvaient-elles et devaient-elles apporter leur soutien aux étrangers "sans-papiers ", "déboutés du droit d'asile " qui veulent entrer et rester sur le territoire français? Or, en rigueur de terme, c'est à l'État qu'appartient le droit de recevoir sur son territoire l'étranger, qu'il prétende au statut de réfugié ou d'immigrant. Politiquement, le «droit d'accueil », fondé éthiquement sur le respect universel des droits de l'homme 6 , est lié au seul «droit d'asile» qui n'est pas illimité. L'accueil de l'immigrant n'est pas de l'ordre d'un tel droit. Cet accueil est guidé par des exigences et des intérêts discernés par l'État?

5. Une des plus longues grèves, début 1992, a eu lieu dans la paroisse Saint-Joseph des Nations à Paris. Elle a duré six mois. Elle a coûté pendant trois mois deux postes salariés à temps complet du Secours Catholique, cinq à six bénévoles à fort investissement et 600000 francs pour l'hébergement et un premier reclassement des personnes qui ont pu être régularisées.

6. Nous n'ignorons pas que le monde anglophone et francophone d'Amérique du Nord préfère à la formule classique et historique des «droits de l'homme » celle des «droits humains" pour éviter toute connotation sexiste à l'emploi du mot "homme». Le concept est alors décontextualisé de son milieu politique, idéologique et occidental pour privilégier une approche plus « universalisable» à toute personne humaine. Pourtant, s'il s'agit bien de vérifier l'universalité de ces droits, c'est bien à partir de l'expression contingente et relative de l'idéal social donné d'une époque qu'il convient de se prononcer. C'est pourquoi, dans le contexte philosophique et historique français, la restitution précise du concept inaugural et séminal «droits de l'homme " nous semble plus adéquate pour rendre compte de l'enjeu de fondation qui s'est joué dans une généalogie des droits.

7. Du côté des exigences, il y a la requête d'intégration harmonieuse des populations et celle de la sauvegarde du bien commun, d'une identité culturelle et d'une politique respectueuse des valeurs de la démocratie et des droits de l'homme. Du côté des intérêts, il y a tout lieu de privilégier ceux qui pourront participer activement au bien commun. C'est ce qui légitime les privilèges accordés en France à certains étrangers dès lors qu'ils apportent des performances sportives ou des compétences techniques, scientifiques ou économiques. 


\subsection{L'Église se risque à agir au nom de sa foi}

C'est pourtant bien dans ce contexte de foi privatisée par la société séculière que l'Église de France se risque à agir au nom de sa foi. Elle se risque à entrer dans le débat public par les prises de parole publiques du Comité épiscopal des Migrations et du Service national de la Pastorale des Migrants. Le 28 avril 2003, les évêques présidents de Justice et Paix, de la Commission Sociale de l'épiscopat et du Comité Épiscopal des Migrations prenaient position sur le droit d'asile. Ils s'adressaient aux citoyens et aux parlementaires afin que les débats qui devaient avoir lieu à l'Assemblée nationale sur cette révision du droit d'asile honorent mieux le troisième terme de la devise de la République, la fraternité. Pour entrer dans ce débat, les évêques n'avaient pour toute légitimité que leur citoyenneté française. C'est comme simples citoyens, animés par leur foi religieuse, qu'ils ont pris la parole. En insistant sur la fraternité universelle, les évêques invoquaient la valeur républicaine qui pouvait être légitimée par la révélation chrétienne. Le 29 septembre dernier, Monseigneur Jean-Luc Brunin, président du Comité épiscopal des Migrations, invitait l'ensemble des agents de la pastorale des migrants en France « à prendre toute initiative utile à la sensibilisation des responsables politiques et à la conscientisation d'une opinion publique largement indifférente à ces questions » $(2004,179)$ et il précisait:

Si l'Église se doit d'intervenir dans le débat qui s'ouvre, elle ne le fait pas pour faire la leçon aux responsables politiques, ni pour légiférer à la place du législateur. Elle le fait à partir de la tradition croyante dont elle est porteuse et au nom de l'expérience vécue par de nombreux chrétiens engagés auprès des migrants dans des associations humanitaires et des organisations de solidarité, confessionnelles ou non. L'Église souhaite, comme les évêques le rappelaient déjà dans leur Lettre aux catholiques de France en 1996, participer loyalement au débat sur tout ce qui contribue au vivre-ensemble. (181)

Enfin, si l'Église se risque à une parole libre sur la présence des étrangers en France, ce n'est pas seulement pour être fidèle aux valeurs de sa tradition. Elle s'y risque parce que c'est son identité et sa mission qui sont en cause dans l'accueil de l'étranger. En faisant place à l'étranger, il ne s'agit pas seulement de mettre en œuvre une éthique de la solidarité à la suite du Christ qui s'est fait proche des petits, des pauvres et des exclus. Cette exigence vigoureuse ne s'apparente pas à une simple obligation morale qui découlerait de la foi, elle constitue une donnée fondamentale de l'identité même de la foi. C'est là sans doute un des points d'argumentation le plus original et le plus vigoureux du texte du Comité épiscopal des Migrations sur Un 
peuple en devenir (1995). Il s'agit plus fondamentalement de vivre et de comprendre la "catholicité » de l'Église confessée dans le Credo. L'accueil de l'étranger est une véritable clé pour ouvrir à l'intelligence du principe catholique du christianisme. Cette catholicité se décline dès l'origine comme communion universelle et comme évangélisation de tous les peuples.

L'Église se risque aussi par ses actes, lorsque de nombreux chrétiens engagés auprès des migrants dans des associations humanitaires et des organisations de solidarité, confessionnelles ou non, soutiennent, encouragent et accueillent les «demandeurs d'asile », les «sans-papiers » et les déboutés du droit d'asile qui attendent sur le territoire français ou qui ne peuvent pas retourner chez eux ${ }^{8}$. Au regard de la loi qui veut qu'on reconduise aux frontières ces étrangers "sans-droits ", certains chrétiens entrent dans l'illégalité et la "désobéissance civile" au nom même de la fidélité à leur foi qui leur commande d'aimer et de servir Dieu dans les plus petits des frères.

\subsection{Un manque de théorisation commune pour rendre compte de cet engagement}

Or, cet engagement de l'Église au nom de la foi est largement controversé tant à l'intérieur de l'Église qu'à l'extérieur. Ad intra, les catholiques sont divisés entre ceux qui pensent que la foi leur commande le devoir de solidarité envers les plus pauvres quels qu'ils soient, et les autres qui, au nom de la distinction entre le spirituel et le temporel, refusent l'entrée de l'Église en politique. Ce serait pour ces derniers soit tomber dans l'idéologie politique et la manipulation d'un tel discours éthique, soit ne pas respecter le principe de laïcité. Ad extra, du côté de l'opinion publique, certains s'enflamment et s'indignent lors des expulsions de clandestins des églises occupées. Pour eux, c'est comme si la communauté ecclésiale avait failli à son droit d'asile et à son éthique de solidarité vis-à-vis des étrangers. Les clandestins, la plupart du temps non-chrétiens, attendent de bénéficier du

8. De nombreux exemples pourraient être signalés par les acteurs du Secours Catholique tel le soutien apporté aux migrants illégaux de la région de Sangatte de 2000 à 2002 et à Nanterre en 1993 où 600 personnes tziganes de Roumanie, installées sur un terrain vague et n'appartenant pas aux catégories pouvant bénéficier du droit d'asile, étaient menacées d'expulsion en plein hiver par la police. Pour éviter la violence des affrontements et trouver la médiation des négociations, le Secours Catholique s'est interposé et a joué la protection des hommes en fuite.

9. Sur la négation juridique de l'asile religieux depuis la loi du 9 décembre 1905, voir Ségur 1995. 
devoir d'accueil étroitement lié aux valeurs partagées par la communauté de foi chrétienne, sans se rendre compte qu'au sein de la société civile pluraliste ces valeurs sont facultatives et n'ont aucune puissance normative. Du côté du monde politique et des autorités civiles on se méfie, voire on s'insurge contre l'irresponsabilité d'une Église qui entre sur le terrain de l'illégalité. Au mieux, on reconnaît qu'il peut exister une opposition entre l'éthique de responsabilité du gouvernement qui est de reconduire les clandestins à la frontière et l'éthique de conviction des chrétiens qui est de prêter assistance au frère en détresse. En plus de toutes ces raisons avouées publiquement, il ne faudrait pas oublier, sous peine de naïveté, les motifs inavouables qui reposent la plupart du temps sur des facteurs psychologiques: refus de toute autorité normative ou peur de l'autre, tout particulièrement de l'étranger.

Finalement, dans un contexte de peur qui stimule les replis identitaires, exhorter à l'accueil de l'étranger au nom de la foi évangélique, comme le fait la pastorale des migrants, peut être interprété comme une provocation politique. Mais ne pas les accueillir semble relever du contre-témoignage de cette même foi. Quoi qu'elle fasse, aux yeux de l'opinion publique, l'Église semble n'avoir de choix qu'entre la «désobéissance civile» ou la culpabilité à l'égard d'un silence ou d'une passivité qui seraient contraires à l'Évangile. Quoi qu'elle dise, son discours reste largement incompris. Mais le problème de fond pour la théologienne que je suis est que l'Église ne semble pas avoir de modèle théorique unanimement acceptable pour justifier combien sa foi peut être une véritable ressource pour le vivre-ensemble.

Cette difficulté nous met au cœur des débats contemporains de la théologie morale. Comprendre adéquatement qu'un agir chrétien ne peut pas être séparé de ses racines théologales et que la foi, comprise dans sa force d'éclairage et de renouvellement de la vie, ne saurait être purement et simplement privatisée relève de la tâche de cette discipline. En revanche, il n'est pas évident que les différents modèles disponibles dans les débats postconciliaires aient tous la même pertinence heuristique pour décliner cet intégralisme de la foi et pour éclairer le type de rapport Église-monde possible dans le contexte de la postmodernité.

\section{Une vision intégrale faible de la foi : la foi «âme de l'engagement » et l'autosécularisation de l'Église}

Le premier modèle qui s'est attaché à rendre compte de la foi comme ressource pour une Église en voie de sécularisation a été celui de l'éthique 
autonome ${ }^{10}$. C'est lui qui, juste après le concile de Vatican II, a permis à l'Église de se comprendre en régime de modernité. Pleinement en rupture avec une vision intransigeante des rapports Église-monde, il soulignait la nécessaire distinction entre foi et politique et entre temporel et spirituel. Ce faisant, ce modèle, en revendiquant l'apport précieux de la foi comme âme de l'engagement dans le monde, n'abandonnait pas la prétention intégrale de la foi.

Le modèle de l'éthique autonome correspond aux positions théorisées par Jacques Maritain dans son livre L'humanisme intégral (1936). Avec la distinction entre le plan temporel où le croyant agit en tant qu'homme et le plan spirituel où celui-ci agit comme chrétien, Maritain allait donner des clés théoriques pour penser un impact pluriel de la foi des chrétiens dans le champ social et politique par médiation des consciences individuelles. Il allait donner une grande chance au ralliement des catholiques à la défense des droits de l'homme en soulignant que la reconnaissance et la formulation de ces droits pouvaient faire l'objet d'une entente pratique entre croyants et incroyants alors même que l'accord rationnel sur leur fondement pourtant nécessaire devenait impossible, voire insurmontable. Au croyant de fonder en conscience l'éminente dignité de tout homme à partir de sa foi. C'est le paradoxe de l'intégralisme de la foi chrétienne que de prétendre asseoir la valeur universelle de tous les êtres humains sur un fondement particulier: celui de la Révélation. Sur le plan éthique, cette option faisait de la morale des chrétiens une éthique rationnelle «informée» par la foi ${ }^{11}$.

10. Ce courant théologique est largement identifié aux travaux d'Alfons Auer, Josef Fuchs, Bruno Schüller, Charles Curran et Richard McCormick, même si chaque théologien a sa manière propre de répondre à la question de la spécificité de la morale chrétienne. Chacun invite à lire la sécularisation non comme une épreuve, mais comme une chance pour la morale chrétienne appelée à défendre et à promouvoir l'humanité de l'être humain.

11. On peut retenir le titre de l'ouvrage de Richard M. Gula (1989) comme exemplaire de la compréhension des débats post-Vatican II en théologie morale catholique: Reason Informed by Faith. Foundations of Catholic Morality. Notons qu'avec cette définition large et générale d'une "éthique rationnelle informée par la foi », il n'est absolument pas évident de savoir comment se fait cette "information ", et encore moins à partir de quel type d'articulation la foi peut-elle «informer» le raisonnement éthique rationnel. Cette définition ne dit pas non plus comment comprendre la foi. S'agit-il de la foi (fides quae) comme adhésion à des contenus, ceux du message chrétien et/ou de la doctrine? Ou bien de la foi comme expérience de la rencontre de Dieu qui engage le croyant à suivre le Christ (fides qua) ? En fait la question n'a pas été clarifiée. 


\subsection{Force de l'éthique autonome pour une Église dans la sécularisation}

La grandeur du modèle de l'éthique autonome, c'était son aptitude à penser «l'expérience commune éthique » entre croyants et non-croyants et de souligner l'universalité de l'éthique chrétienne, comme l'écrivait Xavier Thévenot, lorsque «tout ce qui se commande au nom du Dieu de JésusChrist doit pouvoir se justifier du point de vue de la vérité de l'homme, et tout ce qui est prescrit par la raison droite doit pouvoir montrer sa cohérence avec la vérité de la foi chrétienne» $(1992,15)$. Dans les débats entre théologiens, ce côté théologal de l'éthique autonome n'a pas toujours été vu avec justice ${ }^{12}$. En assimilant ce concept d'autonomie, ces théologiens n'avaient pas à invoquer la révélation pour justifier leurs principes lorsqu'ils se retrouvaient au coude-à-coude avec ceux qui ne partageaient pas leur foi. Les normes morales n'ont pas besoin de l'autorité de leur fondement théologique pour être rationnellement démontrables. Éthique universelle, que tout homme peut découvrir par la raison, l'éthique autonome reconnaissait alors que le caractère chrétien de la morale n'était pas à découvrir dans un contenu normatif spécifique ${ }^{13}$, mais plutôt dans le fait que la foi réinscrit les motivations éthiques dans sa propre logique ${ }^{14}$. On vérifie encore une fois, par cette conception du rôle de la foi, la rupture caractéristique du catholicisme postconciliaire avec l'intransigeantisme. L'éthique autonome se laisse interroger par les questions de l'homme

12. On leur a reproché d'avoir consacré l'œuvre de la sécularisation en promouvant le concept d'autonomie de Kant, alors même que chez cet auteur, comme l'a bien démontré François Marty, c'est de l'intérieur même de l'acte de décision morale que se fait le passage à la question théologique (voir Marty 2002).

13. On trouve cette position exprimée tout particulièrement chez Joseph Fuchs:

Notre réponse à la question posée au sujet du caractère chrétien de la morale catégoriale des chrétiens, c'est-à-dire du comportement concret, s'énonce fondamentalement de la manière suivante. Si nous faisons abstraction de l'élément décisif et essentiel de la moralité chrétienne, de l'intentionnalité chrétienne (comme aspect transcendantal), la morale chrétienne est fondamentalement et essentiellement humaine dans sa détermination catégoriale et sa matérialité. C'est donc une morale d'authentique humanité. (1973)

On trouverait des positions similaires avec des nuances propres à chaque auteur, chez F. Böckle, B. Schüller et J.-M. Aubert.

14. C'était clairement la position adoptée par le philosophe chrétien Jean Lacroix lorsqu'il dit qu'avec la foi chrétienne «la morale humaine n'est aucunement modifiée. Elle conserve ses difficultés et ses espoirs. Elle est seulement ouverte et, suivant un mot de Ricœur, interpellée. Elle reste la même, et cependant reçoit une nouvelle dimension, verticale. Le christianisme ne transforme pas la morale, il la situe» $(1966,115)$. 
moderne et se laisse toucher par les valeurs et principes de l'ethos moderne tout en laissant à la foi son pouvoir d'inspirer les réponses aux défis éthiques rencontrés.

Sans apporter des solutions spécifiques, la foi peut donner un contexte dans lequel vivre sa vie éthique, une intentionnalité religieuse spécifique, que ce soit la vision béatifique, une finalité propre - celle de faire la volonté de Dieu (Schüller 1971, 106) —, ou encore un éclairage particulier sur la vie et le monde capable de conférer un sens achevé à la décision éthique. Pour exprimer cela autrement, la foi conserve une signification historique, présente, en ce qu'elle est une forme, un langage, un corps d'expression, une symbolique qui s'adresse au cœur du croyant et lui permet de fonder le «sens" des valeurs et des normes éthiques auxquelles il se réfère pour mener sa vie éthiquement. Ce qui laisse supposer que la foi peut fonctionner dans cette éthique autonome comme venant conférer à l'agir éthique une «plénitude» de sens. Ainsi, défendre la dignité de tout être humain à la suite du Christ, entraîne à voir que, pour le croyant, la vérité de l'humain est référée à une figure d'humanité à laquelle nous sommes appelés par Dieu en Jésus Christ. Le théologien allemand Dietmar Mieth écrivait à ce sujet que l'originalité chrétienne d'une telle éthique:

... réside plutôt dans l'exigence décisive dont elle témoigne à partir du contexte de l'annonce du salut de l'homme et qu'elle porte au cœur des significations humaines de l'existence et dans les indications normatives qui leur correspondent. La pratique concrète de l'amour chrétien du prochain ne s'affronte ainsi pas uniquement au «texte» que représente la situation de détresse à laquelle il s'agit de vouer ses forces pour la transformer, mais également au «contexte» de la signification du salut. $(1978,102)$

\subsection{Un modèle éclairant pour comprendre des manières encore actuelles de se situer dans l'engagement sociopolitique}

Lorsque les évêques de France entrent dans le débat public sur l'accueil de l'étranger comme simples citoyens, ils demeurent en partie façonnés par ce modèle qui accepte que l'Église institution ne pèse pas directement sur la sphère politique, mais que des croyants puissent donner une inspiration chrétienne à la vie démocratique dès lors qu'on en respecte les lois. Leur appel à la mise en œuvre du droit d'accueil de l'étranger à partir d'une valeur unanimement reconnue dans l'espace public, la fraternité, est typique de ce modèle autonome. La foi motive la prise de parole, elle légitime en profondeur le non-arbitraire de cette valeur par l'appel à la fraternité en Christ. 
Mais la foi n'est pas obligée de se dire explicitement dans la prise de parole des chrétiens lorsque celle-ci s'inscrit dans le débat public.

Dans l'ensemble, bon nombre de militants chrétiens engagés dans le monde associatif non-confessionnel et qui y défendent la dignité de toute personne humaine appartiennent aussi à ce modèle. Ils revendiquent cette défense de l'humain au sein de la société civile comme le critère de ce qui est chrétien. Mais, ils n'ont pas besoin que ceci soit identifié par la société. Ils acceptent sous prétexte de sécularité, d'ouverture et de participation au monde de consentir à "l'aphasie de la foi en matière éthique " (Manaranche $1968,8)$ tout en conservant la plupart du temps en leur cœur un fidéisme muet. Pourtant, c'est bien la foi chrétienne qui demeure l'âme des motivations de leur engagement social ou politique.

Cette manière de voir est venue pour une grande part, dans le champ catholique, d'un souci, exprimé par le concile de Vatican II, d'un appel au " témoignage d'une foi vivante et adulte » dont la «fécondité doit se manifester en pénétrant toute la vie des croyants, y compris leur vie profane, et en les entraînant à la justice et à l'amour, surtout au bénéfice des déshérités " (Gaudium et Spes, $\mathbb{\$} 21,5$; voir Concile de Vatican II 1965). En entrant dans cette stratégie de "participation au monde ", il n'était plus possible de répondre par des théories ou des protestations dogmatisantes aux questions que posaient les faits sociaux. On prenait conscience que la participation au monde conditionne en partie l'intelligence de la foi ${ }^{15}$. La tendance a été alors de lier foi et engagement social par le biais d'une simple relecture opérée dans l'après-coup. Cette relecture donnait un sens à l'engagement des militants, mais elle ne permettait plus de mesurer la portée pratique de la foi en tant qu'elle détermine des engagements et une organisation originale de la vie sociale.

\subsection{Limites de cette éthique autonome}

Mais finalement, tout en prétendant sauvegarder la réalité de la foi et son impact en éthique, l'éthique autonome peut la compromettre en en faisant une détermination adventice venant s'ajouter de l'extérieur à ce que toute

15. Il fallait, comme l'écrivait Michel de Certeau:

... s'enfoncer dans les questions pour y déceler non seulement une situation de fait mais une situation de la foi liée à la condition historique de notre relation avec le Dieu véritable. Tel est le témoignage attendu des chrétiens invités à s'engager délibérément dans les tâches humaines, d'une manière libre et responsable, pour y faire le type d'expérience dont Vatican II leur indique déjà la signification spirituelle. $(1966,531)$ 
éthique séculière peut découvrir en raison. L'humain et le chrétien deviennent éthiquement indiscernables. Cette solution a eu sa force dans une culture encore chrétienne. Dans un monde où les pratiques chrétiennes s'étaient sédimentées progressivement dans l'ethos ambiant, il n'était pas difficile de voir que bien des incroyants consonnaient avec les exigences éthiques chrétiennes ou que celles-ci ne faisaient qu'exprimer une même conscience de l'humain. Mais aujourd'hui, dans une société postmoderne pluraliste où les sujets sont fragilisés par l'anomie ambiante, un tel modèle ne permet pas d'assurer que la raison communicationnelle présidant à la sélection des arguments admis dans l'espace public pour l'adoption des normes ne se trompe pas. Que devient alors le concept de dignité de la personne humaine puisqu'il n'y a plus de lien entre normes et valeurs?

En effet, si les valeurs communes, éprouvées historiquement, ne manquent pas $^{16}$, les ordres axiologiques de la culture pluraliste sont devenus libres de toute légitimation proprement religieuse ${ }^{17}$, voire de légitimation philosophique unanime ${ }^{18}$. Parce que les valeurs sont désormais détachées de tout fondement unitaire, elles peuvent être légitimées de façons plurielles et sont remises à la conviction privée. Mais ces légitimations plurielles dévoilent que, sous l'accord tacite de leur reconnaissance sociale, quand elle existe comme dans le cas de la défense de la dignité humaine, l'apparente unanimité des valeurs cache des anthropologies différentes et un contenu

16. Les débats en bioéthique montrent qu'il y a un noyau dur de valeurs consensuelles qui correspondent à l'ethos des droits de l'homme (voir Fagot-Largeault 1992).

17. Voir Morin 1990 pour une vision nuancée et complexe de l'histoire des valeurs européennes au sein d'une dialogique culturelle réactivée à chaque époque entre les influences gréco-latine, judéo-chrétienne et de la Raison moderne.

18. Ainsi la Déclaration universelle des droits de l'Homme de 1948 n'explicite plus aucun fondement, alors que celle de 1789 reconnaissait encore le «droit naturel » comme légitimation du «non arbitraire » de la déclaration. En 1948, l'obligation éthique de respecter la personne est à la mesure de ce dont il est fait mémoire: l'expérience historique d'un régime de mort et de barbarie qui correspond au déni de la dignité de tout être humain. C'est par la médiation de l'expérience négative et de sa mémoire que l'humanité entend fonder les droits de l'homme sur la «foi» en la dignité de la personne humaine. S'ouvre là un moyen d'attester l'humain dans son universalité sans se référer à un fondement transcendant. La reconnaissance des droits de l'homme se fait indirectement par l'expérience même de leur déni au cœur de la barbarie, du génocide et de la terreur. Il s'agit là d'un jugement moral de la sagesse pratique qui, sur fond d'horreur historique, discerne les chemins qui conduisent à la vie et ceux qui conduisent à la mort. 
prescriptif différent ${ }^{19}$. Le pluralisme éthique doublé d'un pluralisme anthropologique, parce qu'il se pense sur le plan d'une raison communicationnelle et sur le plan démocratique, signe alors l'impossibilité de désigner ce que peut être absolument l'humain pour notre société. Dans ce cas, où le rapport à la confession de foi n'est pas déterminant pour le contenu normatif de l'éthique, la tradition éthique chrétienne n'est plus, pour parler comme Jean-Marc Ferry, qu'un « archivage quelque peu crypté des intuitions éthiques les plus profondes de l'humanité » $(2002,13)$. Au bout du compte, le paradigme de l'éthique autonome n'arrive pas à redonner une pertinence culturelle à la foi chrétienne en tant que contribution originale au débat pluraliste des éthiques, puisque ce qui triomphe, c'est une sagesse commune ou une rationalité scientifique.

Critiqué parce qu'il a correspondu de fait à une "autosécularisation » de l'Église, ce modèle, où l'intégralisme de la foi se joue sous un mode faible, demande d'être dépassé au nom même de la consistance de l'éthique dans une société pluraliste postmoderne. Quand le consensus public sur les valeurs ne permet pas de dire véritablement et unanimement ce qu'est l'humain, la foi chrétienne ne peut pas être ravalée au rang d'une simple opinion entièrement contextualisée. La vérité de l'humain, confessée dans la foi, relative à une expérience, à une parole et à une relation ne saurait être tenue pour relative par le croyant. Elle demande d'être attestée, parce qu'elle est reconnaissance d'une filiation croyante qui préserve le croyant du fantasme d'être à lui-même sa propre origine. D'où le nouvel impératif pour l'Église, de témoigner et de prendre la parole dans un nouveau procès de socialité.

\section{Une vision intégrale forte de la foi pour une Église minoritaire: la foi comme règle de vie et style de vie}

L'éthique de la foi et l'éthique communautarienne de la vertu sont les deux modèles qui correspondent à cette attestation. Tous deux partagent une vision intégrale de la foi, cette fois-ci forte, refusant la privatisation de la foi de l'Église. Mais c'est leur appréciation du temps présent qui les différencie. Le premier modèle me semble encore se penser en fonction d'un contexte moderne, alors que le second se situe explicitement dans le contexte de la postmodernité.

19. Le cas exemplaire peut être le débat autour du respect de la personne humaine en fin de vie avec l'option de l'euthanasie ou celle des soins palliatifs. Chacun défend dans le débat public des visions de l'homme fort différentes à travers ces deux normes d'action. 


\subsection{Le modèle de l'éthique de la foi: la foi comme règle de vie}

Le modèle de l'éthique de la foi est né en réaction à l'interprétation du rapport foi-éthique du modèle autonome qui venait miner le contenu spécifique d'un comportement chrétien et le rôle social de la foi (Ratzinger et Delhaye 1979). Il s'agissait alors, pour les chefs de file de ce courant tels que Philippe Delhaye et Joseph Ratzinger, d'insister sur la contribution spécifique de la foi chrétienne, capable d'instituer de la loi et des repères éthiques pour des libertés qui s'engagent à la suite du Christ. Contre le danger d'une autosécularisation de l'Église qui risquait de ne plus dire autre chose que ce que le monde pouvait tenir, il fallait revenir à une vision intégrale de la foi permettant de rendre compte que la personne que vise l'acte de foi est une personne unifiée et non clivée dans une pluralité de rôles indépendants les uns des autres. La foi ne saurait être cantonnée dans le culte et la prière. Elle comporte des exigences éthiques et sociales, parce qu'elle concerne la vie de l'homme dans toutes ses dimensions. Contre une rationalisation de l'éthique, il fallait revenir à ses sources scripturaires, car la foi évangélique a toujours été, à travers les cultures traversées, source de discernement des pratiques.

La thèse principale de ce courant, c'est que certaines solutions éthiques ne peuvent être comprises en dehors du cadre de la foi au Christ. Car la foi, comprise selon la formule de Lumen Gentium, $\$ 25$ : "fides credenda et moribus applicanda", n'est pas seulement une adhésion de l'esprit à Dieu. Elle est une règle de vie, une exigence évangélique qui s'étend aux actions et à la praxis. Celui qui est informé par la méditation des Écritures, la pratique liturgique et la vie communautaire peut trouver dans l'événement ce que la vie de la foi doit lui inspirer à ce moment-là. Comme l'a écrit H.U. von Balthasar, c'est «Jésus-Christ » lui-même comme révélateur de l'amour divin trinitaire qui est la norme. "C'est du point de vue du Christ, c'est-àdire de la foi, que le chrétien arrêtera les options profondes de sa vie.» $(1979,73)$ Ce faisant, «Les valeurs fondamentales de l'homme, qu'elle connaît en portant le regard sur l'exemple de Jésus-Christ, la foi les soustrait à toute manipulation» (Ratzinger 1979, 129).

On mesure la force éthique de cette posture théologique. Face à la raison communicationnelle de l'éthique procédurale, incapable de lier une norme à une valeur éthique sûre, l'éthique de la foi permet de rétablir le lien. Là où il n'y a plus d'unanimité, elle souligne que tous les grands défis éthiques d'aujourd'hui - euthanasie, clonage, avortement, développement 
durable, respect des droits humains - ne sauraient être traités sans l'éclairage des convictions religieuses qui considèrent l'homme dans sa finitude, ses peurs, ses angoisses et ses espoirs. Avec un tel modèle, une définition de la «vie bonne» en soi est alors possible. Une dissidence morale dans les grands débats éthiques redevient pensable, puisque la foi dessine un certain visage de l'humanité référé à l'icône du Christ. Alors que la religion, selon le schéma évolutionniste classique de la Modernité, est censée disparaître de la scène publique, l'éthique de la foi sait la nécessité de recouvrer les richesses de la tradition ecclésiale non seulement pour maintenir une identité chrétienne, mais aussi pour assurer une vision de l'humain non sujette au relativisme.

Lorsque Monseigneur Brunin revendique d'intervenir dans le débat public autour de l'accueil de l'étranger, il le fait dans cet esprit, au nom de la tradition de la foi chrétienne qui commande d'aimer et de servir Dieu dans le frère, de vêtir les pauvres, de nourrir les affamés et de libérer les prisonniers. Évêque de la jeune génération, il n'appartient plus au modèle autonome de ses prédécesseurs. Se référer à la tradition, c'est alors défendre la mémoire prophétique de Jésus Christ. Une mémoire qui invite à mettre en œuvre la foi caritative, véridiction de l'amour de Dieu servi dans les frères à la manière de Jésus.

La critique majeure qu'on peut adresser à ce modèle, ce n'est pas le risque d'intransigeantisme et de dérive conservatrice que certains théologiens de tendance libérale ont cru y discerner. C'est plutôt sa difficulté à désigner le présent et les contraintes qu'il impose au témoignage de la foi. Finalement, qu'il s'agisse de l'éthique autonome ou de l'éthique de la foi, on ne se préoccupe guère de savoir s'il y a des sujets capables de responsabilité et de discernement. En se prononçant massivement sur la vérité de la norme, l'éthique de la foi fait comme si l'on pouvait présupposer qu'existent des sujets suffisamment construits et autonomes pour comprendre et saisir cette vérité qui était en jeu, alors que dans un contexte postmoderne, ce qui manque, ce sont les sujets pour la mettre en œuvre. À l'heure où nous diagnostiquons toute la fragilité des libertés qui doivent apprendre à se repérer dans le pluralisme ambiant et toute la place de l'individu dans l'inscription du sens, il est important, si l'on veut penser de manière réaliste la foi comme une ressource pour l'agir, de prendre en compte le problème que pose la généalogie du sujet moral au cœur de la communauté chrétienne. C'est tout l'intérêt du dernier modèle, celui de l'éthique communautarienne de la vertu. 


\section{L'éthique communautarienne de la vertu: la foi comme style de vie}

Ce modèle, qui se situe dans la lignée confessante et attestataire du précédent, le radicalise. Il s'agit bien d'une éthique de la foi dont le rôle est de rappeler que la vie morale n'est pas seulement la résolution de cas concrets, mais bien plus une question de la configuration de la vraie vie ${ }^{20}$ à la suite du Christ. Représenté emblématiquement par le travail du théologien protestant nord-américain Stanley Hauerwas, ce modèle a le mérite de vouloir penser et promouvoir une éthique confessante enracinée sur la dimension communautaire. La grande question que Hauerwas reprend de son maître Gustafson est la suivante: "Qu'est-ce que cela change pour la vie éthique lorsqu'on la pense théologiquement? » La foi engendre-t-elle un modèle de vie, des attitudes et des comportements? Aux yeux de Hauerwas, ce qu'il est important à discerner, c'est que la vision chrétienne du monde est liée à un type de «caractère ", de personnalité, elle-même dépendante des récits portés par la tradition. Car, de son dialogue avec le philosophe MacIntyre, Hauerwas sort convaincu que mener sa vie morale ne relève pas que d'une aptitude à penser clairement et à faire des choix rationnels. C'est une manière de voir le monde et d'être-au-monde. La question fondamentale pour envisager la moralité et le façonnement d'une personnalité, d'un sujet, est donc de savoir de quelle histoire sa vie fait partie. À quelle communauté de souvenir, à quelle pratique engagée, et à quel dessein a-til part? Car des sociétés différentes produisent des projets de vie différents, des visions de vie bonne dissemblables, des types de sujets représentatifs divergents et des vertus distinctes. On comprend alors que le type de tradition et de communauté où le sujet reçoit son identité fera toute la différence du caractère. D'où l'insistance mise sur la nécessaire position contre-culturelle de l'éthique communautaire face au monde. Une éthique critique et de résistance, parce que fondée sur le récit et la mémoire dangereuse de la Passion et de la résurrection de Jésus Christ.

20. L'expérience des dissidents de l'Est européen dans les années 1970 est un bon exemple de ce rappel, en contexte non religieux. V. Havel et les signataires de la Charte 77 ont montré que leur indignation face au mensonge de la société communiste n'était pas sans objectivité. Car ce qui pouvait la mouvoir et impliquer les dissidents jusqu'au prix du sang, c'est la capacité du sujet à vivre tout simplement sa vie d'homme en communauté avec d'autres. En effet, cette indignation éthique naît, pour les dissidents de l'Est, à partir de l'expérience historique de l'impossibilité d'assumer toute vie sociale autonome, c'est-à-dire la liberté et la responsabilité de sa propre vie. Et par contraste, ce déni de la «vraie vie» la désigne comme valeur inconditionnelle à respecter. 
Ce modèle "sectarien" ne manque pas de force pour le soutien des libertés éthiques fragiles de la postmodernité. Il insiste sur un point qu'aucun théologien ne devrait pouvoir contester: la nécessité de l'ethos communautaire et de la tradition avec leurs récits et leurs exempla pour former l'identité des chrétiens, tant individuellement que communautairement. Voir la foi comme style de vie, comme force d'unification et de cohérence, parce qu'elle permet au sujet d'échapper au seul débat avec lui-même et au relativisme possible d'une pluralité de références éthiques dans la société, ne manque pas de pertinence. À cela vient s'ajouter de manière paradoxale un service de la société quand la communauté reste fidèle à sa vision spécifique du monde et que, par le témoignage, rendu jusqu'au sacrifice de soi, du service de l'amour, de la justice et de la fraternité, elle peut nourrir les combats de la société civile ${ }^{21}$. Ce modèle ne manque pas de force non plus pour penser que la religion n'est pas réductible à une simple hétéronomie qui s'opposerait à l'autonomie discursive de l'argumentation éthique. La religion, parce qu'elle est portée par des récits, appelle la possibilité de la discursivité. Les illusions libérales de l'universalisme et de la neutralité des éthiques procédurales empêchent de voir que la rationalité éthique n'est jamais libérée de particularisme. Qu'elle soit théorique ou pratique, la rationalité est un concept qui a une histoire. Et la tradition n'est pas forcément l'ennemie de la raison. Dire ou croire cela serait céder à une conception rationaliste et instrumentale de la raison.

Comme les deux précédents modèles, celui-ci nous permet de comprendre certaines manières de se situer dans les communautés chrétiennes et dans la société. Lorsque les étrangers sans droits et sans papiers viennent demander à l'Église refuge et asile en vertu de sa tradition d'accueil, de fait, l’Église se voit attribuer le rôle de communauté prophétique nourricière d'une conception de la défense des droits de l'homme en "excès" sur son application juridique. De même, lorsque des chrétiens ou des communautés

21. Le service de la société a été explicité en Linbeck 2002. Enracinée sur une ecclésiologie sectarienne, ce modèle développe la pertinence sociale d'une communauté lorsqu'elle reste fidèle à sa vision spécifique du monde et à ses valeurs propres. Il en va de la mission de l'Église de témoigner jusqu'au sacrifice du service de l'amour, de la justice et de la fraternité. L'enclave sectarienne de l'Église est alors un lieu d'attestation qui peut nourrir les combats de la société civile. Linbeck va même jusqu'à dire que les sociétés démocratiques séculières peuvent prendre appui sur ces enclaves communautaires dans la mesure où celles-ci encouragent leurs membres «à se préoccuper des autres et non de prérogatives et de droits individuels ", et les poussent "à un sens des responsabilités pour la société au sens large» $(2003,169)$. 
entrent dans la désobéissance civile au nom de leur foi en accueillant des sans-papiers et constituent alors des enclaves dissidentes porteuses d'un certain visage de l'humain, ils témoignent de la fécondité sociale de cette vision intégraliste de la foi.

Mais, là encore ce modèle n'a pas manqué de critiques ${ }^{22}$. Je me contenterai d'en souligner deux qui concernent plus particulièrement notre propos. La première critique à relever concerne la communauté d'appartenance du sujet croyant dans la société postmoderne. À moins de vivre dans un ghetto, le sujet croyant n'appartient jamais à une seule communauté. C'est bien cela le drame des sujets postmodernes: arriver à se repérer dans cette pluralité de sphères de vie. Or, la position communautarienne, qui met en valeur l'appartenance du sujet à une communauté ecclésiale bien déterminée, risque d'oublier que nous sommes «empêtrés dans une pluralité de récits, d'histoires et de traditions » dont il faut pouvoir rendre compte. Dans un tel système, demeure alors ouverte la question proprement éthique du lien entre l'objectivité d'une éthique fondée sur la foi et l'intention de vie du sujet. La deuxième critique concerne le visage sectarien que prend l'Église dans ce modèle communautarien. Si cette éthique narrative de la vertu rend bien compte du spécifique chrétien, et si elle voit très nettement qu'un renouveau de la théologie morale par la tradition de la vertu doit s'engager dans une orthopraxie d'émancipation sociale vraie, elle ne saurait prendre le visage d'une spécificité totalitaire coupée de toute discussion publique. Il ne faut pas oublier que dans la particularité du récit évangélique est inscrit un appel à l'universel.

\section{Conclusion}

Pour conclure, je ferai trois remarques en direction du thème du présent numéro.

Les moralistes qui se sont affrontés dans des débats parfois violents depuis le concile n'ont pas failli à leur responsabilité en prenant pour tâche de défendre que la foi chrétienne rend possible la conduite et la gestion consciente et responsable des questions de société. Les trois modèles examinés s'accordent tous sur ce point. Mais, il faut préciser que le mot «foi » ne saurait s'entendre exclusivement comme un corpus de propositions qu'il

22. Pour n'en citer que quelques-unes adressées à Hauerwas: Lammers 1993, 57-77; Delkeskamp-Hayes 1995, 48-64; Lange 1992, 192-199; Müller 1999, 50-52. 
suffirait de tenir pour vraies. La foi se rapporte à la vie et propose de faire vivre en ce monde ceux qui s'y ouvrent et la pratiquent. Elle est un style de vie à la suite du Christ qui pousse l'humanité à ne pas désespérer de la vie. Voilà pourquoi, une théologie morale responsable devrait toujours pouvoir dialoguer avec sa culture et son temps dans un souci d'ouverture à l'universalité de l'humanité.

Les moralistes qui ont rendu raison de l'intégralisme de la foi dans une société qui risquait de la privatiser n'ont pas manqué à leur responsabilité quand bien même aucun de leur modèle serait pleinement satisfaisant pour nous aujourd'hui. Ils nous laissent la leçon que tout effort théologique correspond à un acte de discernement inscrit dans une époque, dans des problématiques propres correspondant à une culture et à une société données. Ils nous enseignent le devoir de continuer à notre tour de risquer notre propre interprétation.

Dans ce risque, nous ne sommes pas de simples inventeurs. Ceux qui nous ont précédés nous laissent des points d'attention à ne pas négliger si nous voulons honorer les divers publics de la théologie. Trois points forts peuvent être retenus de notre parcours.

- L'exigence éthique est rationnelle et universelle. Dieu ne commande rien d'absurde; nous devons, en principe pouvoir compter sur l'intelligibilité de ce que nous annonçons du côté de ceux auxquels nous nous adressons même s'ils ne sont pas chrétiens. C'est notre devoir de le manifester.

- Nombre d'exigences et de convictions éthiques nous ont été révélées dans le cadre de l'Alliance; notre chemin vers l'éthique n'est généralement pas celui de la libre élaboration théorique. La vulnérabilité à l'exigence éthique, pour rationnelle qu'elle soit, est le plus souvent créée en nous par la foi et se rend connaissable dans le cadre de la cohérence de la révélation. Le chrétien doit donc s'attendre à ne pas être compris de tous.

- Dans un monde postmoderne au pluralisme exacerbé, la possibilité d'un agir conforme à l'Évangile doit être suscitée dans le cadre d'une communauté qui forme des sujets et qui sait qu'il est déraisonnable de compter sur les seules forces d'un raisonnement pour convaincre chacun là où son existence est mise en jeu au plus profond. C'est par une symbolique, une rhétorique, une herméneutique des Écritures, etc., en s'appuyant sur la fraternité d'une communauté que le sujet 
postmoderne fragilisé pourra se laisser atteindre par la beauté de la vérité.

C'est au prix de l'articulation entre ces trois points forts que le moraliste pourra penser la foi chrétienne comme ressource pour vivre les questions sociales de notre temps et exercera son service de la vie de l'Église et de la mission auprès de nos contemporains.

\section{Références}

Berten, A., P. Da Silveira et H. Pourtois, dir. (1997), Libéraux et communautariens, Paris, Presses universitaires de France (Éthique et philosophie morale 1 ).

Brunin, J.-L. (2004), «Au cœur d'une politique migratoire, le primat de l'humain » / message aux membres du réseau de la Pastorale des Migrants, Documentation catholique, 15 février 2004, n 2308, p. 179 180 .

Certeau, M. de (1966), «De la participation au discernement. Tâche chrétienne après Vatican II ", Christus, 52, p. 518-537.

Comité episcopal des Migrations (1995), Un peuple en devenir. L’Église et les migrants, Paris, Atelier.

Concile de Vatican II (1965), Constitution pastorale Gaudium et Spes sur l'Église dans le monde de ce temps, accessible sur le Web à : <www.vatican. va/archive/hist_councils/ii_vatican_council/documents/vat-ii_cons_ 19651207_gaudium-et-spes_fr.html>.

Conférence des Évêques de France (1994), Proposer la foi dans la société actuelle, $\mathrm{t}$. I / rapport présenté par $\mathrm{M}^{\mathrm{gr}} \mathrm{C}$. Dagens à l'assemblée plénière de Lourdes 1994, Paris, Cerf (Documents des Églises — Public large).

- (1996), Proposer la foi dans la société actuelle, t. III : Lettre aux catholiques de France / rapport dirigé par $\mathrm{M}^{\mathrm{gr}} \mathrm{C}$. Dagens et adopté par l'assemblée plénière des évêques de France, Paris, Cerf (Documents des Églises - Public large).

Delkeskamp-Hayes, C. (1995), « Towards a Non-Ecumenical Interchange: Engelhardt, Hauerwas and Ramsey on Christian Bioethics ", Christian Bioethics, 1/1, p. 48-64.

DonéGANi, J.-M. (1999), «Une désignation sociologique du présent comme chance », dans H.-J. GageY et D. Villepelet, Sur la proposition de la foi, Paris, Atelier, p. 39-58.

Doré, J. (2003), La grâce de croire, t. II : La foi, Paris, Atelier. 
Fagot-Largeault, A. (1992), "La réflexion philosophique en bioéthique », dans M.-H. Parizeau, Les fondements de la bioéthique, Bruxelles, De Boeck Université, p. 13-17.

Ferry, J.-M. (2002), Valeurs et normes. La question de l'éthique, Bruxelles, Éditions de l'Université de Bruxelles.

Fuchs, J. (1973), Existe-t-il une morale chrétienne?, Gembloux, Duculot.

Gula, R.M. (1989), Reason Informed by Faith. Foundations of Catholic Morality, New York, Paulist.

Habermas, J. (1994), «Israel oder Athen: Wem gehört die anamnetische Vernunft? Johann Baptist Metz zur Einheit in der multikulturellen Vielfalt », dans J.-B. Metz, Diagnosen der Zeit, Düsseldorf, Patmos, p. 51-64.

LACRoix, J. (1966), "Morale, métaphysique et religion ", Recherches et débats, 55, p. 102-118.

Lammers, S.E. (1993), "On Stanley Hauerwas. Theology, Medical Ethics and the Church ", dans A. Verhey et S.E. Lammers, dir., Theological Voices in Medical Ethics, Grand Rapids, Eerdmans, p. 57-77.

LAnge, D. (1992), Ethik in evangelischer Perspektive, Göttingen, Vandenhoeck \& Ruprecht.

Linbeck, G. (2002), «Ecumenism and the Future of Belief » [1968], dans J. Buckley, dir., The Church in a Postliberal Age, Londres, SCM Press, p. $91-105$.

— (2003) [1984], La nature des doctrines, Paris, Van Dieren.

Manaranche, A. (1968), Y-a-t-il une éthique sociale chrétienne?, Paris, Seuil.

Maritain, J. (1936), L'humanisme intégral. Problèmes temporels et spirituels d'une nouvelle chrétienté, Paris, Aubier.

MARTy, F. (2002), «Dieu aux frontières de l'éthique », dans le collectif Dieu a-t-il sa place dans l'éthique? I actes de la journée d'étude «Dieu a-t-il sa place dans l'éthique?» organisée par la Fondation Ostad Elahi Éthique et solidarité humaine, Paris, 28 octobre 2000, Paris, L'Harmattan (Logiques du Spirituel), p. 13-25.

Mieth, D. (1978), «Autonomie. Emploi du terme en morale chrétienne fondamentale ", dans C.J. Pinto De Oliveira, dir., Autonomie. Dimensions éthiques de la liberté, Fribourg / Paris, Éditions universitaires de Fribourg / Cerf, p. 85-103. 
Morin, E. (1990), «L'invention de l'Europe», dans Les Cahiers Français, 244: La France et l'Europe, janvier-février, Paris, La documentation française, p. 12-18.

MülleR, D. (1999), L'éthique protestante dans la crise de la modernité, Paris / Genève, Cerf (Passages) / Labor et Fides.

PIE X (s.d.), E supremi apostolatus, dans Actes de S.S. Pie X, Paris, Bonne Presse, vol. 1, p 30-48.

RAtZinger, J. (1979), "Foi, éthique et Magistère », dans J. Ratzinger et P. Delhaye, Principes d'éthique chrétienne, Paris, Lethielleux, p. 103-137.

Ratzinger, J. et P. Delhaye (1979), Principes d'éthique chrétienne, Paris, Lethielleux.

SCHÜller, B. (1971), «Die Bedeutung des natürlichen Sittengesetzes für den Christen ", dans G. TeichtweIER et W. Dreier, dir., Herausforderung und Kritik der Moraltheologie, Würzburg, Echter.

SÉGuR, P. (1995), "Le droit d'asile religieux: un droit moribond ? ", dans le collectif Droit d'asile, devoir d'accueil. VIII ${ }^{e}$ Colloque de la Fondation Jean Rodhain, Lourdes 16-19 novembre 1994, Paris, Desclée de Brouwer, p. 87-122.

Thévenot, X. (1992), Compter sur Dieu. Étude de théologie morale, Paris, Cerf (Recherches morales).

Von Balthasar, H.U. (1979), "Neuf thèses pour une éthique chrétienne ", dans J. Ratzinger et P. Delhaye, Principes d'éthique chrétienne, Paris, Lethielleux.

\section{Résumé}

Pour répondre aux grands défis de la société actuelle, la foi chrétienne peutelle nous aider à inventer, orienter et organiser une vie morale? À la lumière de la confrontation de l'Église de France à la délicate question de l'accueil des étrangers illégaux, l'auteure revisite les trois modèles d'éthique théologique disponibles dans les débats postconciliaires: l'éthique autonome, l'éthique de la foi et l'éthique communautarienne de la vertu. Forces et limites de ces modèles d'implications sociales de la foi supposent la catégorie de l'intégralisme de la foi. 


\section{Abstract}

In response to the great challenges of contemporary society, how can Christian faith help us to invent, direct and organise moral life? In light of the confrontation of the Church of France towards the delicate situation of illegal immigrants, the author reviews three models of theological ethics that emerged during the post-conciliatory debates: autonomous ethics, ethics of faith and community ethics of virtue. The strengths and limitations of these models of social implications of faith imply the category of integralism of faith. 